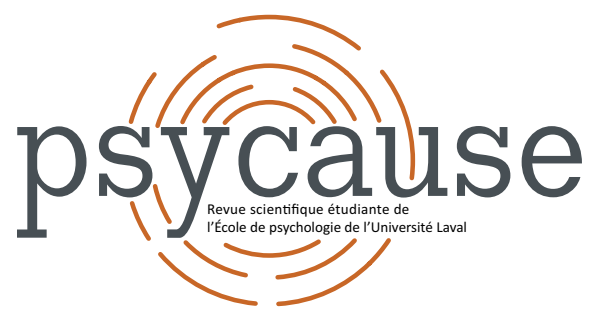

\title{
PSYCAUSE
}

Revue scientifique étudiante de l'École de psychologie de l'Université Laval

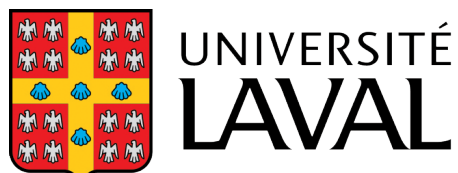

Faculté des sciences sociales École de psychologie

OCTOBRE 2021 - VOL. $11 \mathrm{~N}^{\circ} 2$

\section{VALIDATION INITIALE D'UNE ADAPTATION POUR ENFANTS DE LA SELF-COMPASSION SCALE}

Marjorie SIMARD ${ }^{1 *}$, Trycia MIGNEAULT ${ }^{1}$, Klara BOUCHARD ${ }^{1}$ et Nancie ROULEAU1'-2-3

${ }^{1}$ École de psychologie, Université Laval

${ }^{2}$ MANDALAB

${ }^{3}$ Centre de recherche CERVO

*marjorie.simard.3@ulaval.ca

\section{Pour citer l'article}

Simard, M., Migneault, T., Bouchard, K., \& Rouleau, N. (2021). Validation initiale d'une adaptation pour enfants de la Self-Compassion Scale. Psycause: Revue scientifique étudiante de l'École de psychologie de l'Université Laval, 11(2), 24-25. 
irritable bowel syndrome and fibromyalgia/arthritis and 83(6), 384-385. https://doi.org/10.1159/000365174

in healthy controls. Psychotherapy and Psychosomatics,

\title{
Pour citer l'article
}

Côté, G., Lacombe, L., O’Neill-Readman, L., Massicotte, V., Ivers, H., \& Savard, J. (2021). Perfectionnisme et stratégies d'adaptation: contribution du sexe, de l'âge et du type de cancer. Psycause: Revue scientifique étudiante de l'École de psychologie de l'Université Laval, 11(2), 22-24.

\section{VALIDATION INITIALE D'UNE ADAPTATION POUR ENFANTS DE LA SELF-COMPASSION SCALE}

\author{
Marjorie SIMARD ${ }^{1 *}$, Trycia MIGNEAULT ${ }^{1}$, Klara BOUCHARD ${ }^{1}$ et Nancie ROULEAU ${ }^{1-2-3}$ \\ ${ }^{1}$ École de psychologie, Université Laval, ${ }^{2}$ MANDALAB, ${ }^{3}$ Centre de recherche CERVO \\ *marjorie.simard.3@ulaval.ca
}

\section{Mots-clés : compassion pour soi, mesures, pleine conscience, enfants}

La compassion se définit comme une réponse émotionnelle bienveillante face à la souffrance d'autrui, associée au désir de voir cette souffrance allégée (Gilbert, 2020). La compassion est communément abordée comme étant dirigée vers autrui, mais elle peut être dirigée envers soi. Selon le modèle de Neff (2003), la compassion pour soi (CS) inclut trois composantes. Premièrement, la pleine conscience (PC) est définie comme l'attention portée intentionnellement au moment présent, permettant de percevoir sa propre souffrance. La PC est le contraire de la sur-identification qui consiste à s'immerger dans ses réactions émotionnelles. Deuxièmement, l'humanité commune consiste à concevoir sa souffrance comme indissociable de l'expérience humaine et s'oppose à l'isolement correspondant à la perception qu'une situation vécue est unique à soi. Troisièmement, la bienveillance est l'attitude d'accueil, de non-jugement et de gentillesse envers soi, en réaction à la souffrance vécue. Elle se différencie de l'autocritique. La CS est une habileté importante et son entraînement a démontré une réduction de l'intimidation à l'école (Gonynor, 2016), en plus de contrer l'isolement en raison de la connexion sociale qu'elle procure (Rouleau, 2021).

Afin de mesurer la CS, le principal outil utilisé est la SelfCompassion Scale (SCS) (Neff, 2003). Cet outil a été initialement construit pour les adultes et a été adapté en une version courte, la Self-Compassion Scale-Short Form (SCS-SF; Raes et al., 2011). Toutefois, à ce jour, aucune validation française de la SCS n'a été effectuée et peu d'outils ont été validés pour mesurer la CS chez les enfants. Bien que la CS fait déjà l'objet de quelques interventions en milieu scolaire, aucune étude n'a encore exploré sa trajectoire développementale auprès des enfants. Or, un obstacle à l'avancement de ces connaissances est l'absence d'outils de mesure validés auprès d'enfants. Le MANDALAB (Mindfulness AND
Attention LAB) a donc traduit en français la SCS-SF et l'a adaptée pour être utilisée auprès d'enfants. Cette adaptation est justifiée par les différences développementales sur le plan psychologique, cognitif et social ainsi que par les différences culturelles, notamment le langage des enfants québécois. La présente étude vise à effectuer une première phase de validation pour vérifier les propriétés psychométriques de l'Échelle de Compassion pour Soi pour Enfants (ÉCS-E) chez des enfants québécois francophones de $3^{e}$ et $4^{\mathrm{e}}$ année du primaire.

\section{Méthode}

Les participants ont été recrutés dans le cadre d'une étude plus large du MANDALAB réalisée en 2018 ayant pour objectif d'évaluer l'efficacité de l'entraînement par la pleine conscience (intervention PEACE) sur diverses variables. L'échantillon est formé de 135 enfants âgés de 8 à 11 ans, soit de la $3^{\mathrm{e}}$ et $4^{\mathrm{e}}$ année du primaire. Les enfants ont été répartis en deux groupes: un groupe contrôle actif $(n=56)$ et un groupe intervention (PEACE) $(n=79)$. Avec un niveau alpha fixé à .05 , des analyses de khi-carré ont confirmé l'équivalence des groupes par rapport à la répartition du sexe et du niveau scolaire. Les données descriptives des groupes sont présentées dans le Tableau 1.

Deux traductrices indépendantes ont effectué une traduction inversée de la version courte de la SCS (Raes et al., 2011). Cette version traduite du SCS a été adaptée par le MANDALAB. Pour les 12 énoncés que comprennent I'ÉCS-E, les répondants doivent inscrire à quelle fréquence ils agissent de la façon décrite sur une échelle de Likert allant de 1 («presque jamais») à 5 («presque toujours»). Chaque énoncé évalue un des deux pôles des trois composantes du 
Tableau 1

Données sociodémographiques et statistiques descriptives des participants $(n=135)$

\begin{tabular}{lcccccccccc}
\hline & \multicolumn{4}{c}{ Groupe PEACE-School } & \multicolumn{3}{c}{ Groupe contrôle } \\
\cline { 2 - 9 } Variables & $n$ & $M$ & $E T$ & $\%$ & $n$ & $M$ & $E T$ & $\%$ \\
\hline Sexe & & & & & & & & \\
$\quad$ Garçons & 40 & 35,33 & 8,11 & 50,60 & 30 & 34,97 & 7,82 & 53,60 \\
$\quad$ Filles & 39 & 35,18 & 4,84 & 49,40 & 26 & 34,92 & 8,12 & 46,40 \\
$\quad$ Niveau scolaire & & & & & & & & & \\
$\quad 3^{e}$ année & 30 & 34,53 & 5,80 & 50,60 & 28 & 36,25 & 8,83 & 50 \\
$\quad 4^{e}$ année & 39 & 36 & 7,44 & 49,40 & 28 & 33,64 & 6,73 & 50 & \\
\hline
\end{tabular}

Note. Total de l'ÉCS-E $=60$

modèle de Neff (2003). Un score total sur 60 est calculé en additionnant la réponse à chacun des énoncés.

Un auxiliaire de recherche du laboratoire lisait les énoncés à voix haute et un à la fois, tandis que les enfants remplissaient individuellement le questionnaire en format papier. Les données utilisées dans la présente étude proviennent des réponses données avant la mise en place de l'intervention (semaine 0) et à la toute fin du programme (semaine 9).

\section{Résultats et discussion}

Les résultats supportent une structure à deux facteurs, soit les énoncés correspondant aux composantes et ceux mesurant leurs opposés. Ce résultat se différencie de la structure factorielle retrouvée chez les adultes, indiquant que la CS pourrait se raffiner avec l'âge.

Un alpha de Cronbach de .63 a été obtenu, appuyant une cohérence interne satisfaisante selon Cohen et ses collaborateurs (2008). Seuls les énoncés 6 et 7 , lorsque retirés, augmentaient légèrement l'alpha de Cronbach à 65 . Ce résultat suggère que I'ÉSC-E est conforme en ce qui concerne le construit.

Une corrélation de Pearson positive et modérée entre les scores totaux obtenus aux deux temps de mesure, incluant seulement le groupe contrôle afin d'éviter un effet d'intervention, montre une fidélité test-retest satisfaisante et l'absence d'effet de pratique.
En conclusion, les résultats indiquent que l'ÉCS-E est valide sur le plan de la structure factorielle et du construit chez les garçons et les filles de 8 à 11 ans. Pour la fidélité de l'ÉCS-E, la stabilité a aussi été appuyée. Étant donné qu'une révision des énoncés de l'ÉCS-E devra être effectuée, une deuxième validation de l'échelle sera aussi nécessaire afin de confirmer et détailler ses valeurs psychométriques. Néanmoins, l'entraînement de la CS à l'enfance est une intervention prometteuse méritant des outils qui l'évaluent à sa juste valeur.

\section{Références}

Cohen, L., Manion, L., \& Morrison, K. (2008). Research Methods in Education. In Research Methods in Education. http:// doi.org/10.4324/9781315456539

Gilbert, P. (2020). Compassion: From its evolution to a psychotherapy. Frontiers in Psychology, 11, 3123. http:// doi.org/10.3389/fpsyg.2020.586161

Gonynor, K., A. (2016). Associations among mindfulness, self-compassion, and bullying inearly adolescence [mémoire de maitrise, Université d'État du Colorado]. Mountainscholar. https://mountainscholar.org/bitstream/ handle/10217/176658/Gonynor_colostate_0053N_3691. pdf? sequence $=1 \&$ isAllowed $=y$

Neff, K. D. (2003). The Development and Validation of a Scale to Measure Self-Compassion. Self and Identity, 2(3), 223250. http://doi.org/10.1080/15298860309027

Raes, F., Pommier, E., Neff, K. D., \& Van Gucht, D. (2011). Construction and factorial validation of a short form of the Self-Compassion Scale. Clinical Psychology and Psychotherapy, 18(3), 250-255. http://doi.org/10.1002/cpp.702

Rouleau, N. (2021). PSY 8065 Fondements empiriques et applications cliniques des approches basées sur la pleine conscience [notes de cours]. École de psychologie, Université Laval.

\section{Pour citer l'article}

Simard, M., Migneault, T., Bouchard, K., \& Rouleau, N. (2021). Validation initiale d'une adaptation pour enfants de la SelfCompassion Scale. Psycause: Revue scientifique étudiante de l'École de psychologie de l'Université Laval, 11(2), 24-25. 